Draft Version May 19, 2020

Typeset using $\mathrm{LAT}_{\mathrm{E}} \mathrm{X}$ twocolumn style in AASTeX63

\title{
Nature of Compton-thick Active Galactic Nuclei in "Non-merging" Luminous Infrared Galaxies UGC 2608 and NGC 5135 Revealed with Broadband X-ray Spectroscopy
}

\author{
Satoshi Yamada (iD, ${ }^{1}$ Yoshihiro Ueda (D), ${ }^{1}$ Atsushi Tanimoto $\mathbb{D},{ }^{1}$ Saeko Oda, ${ }^{1}$ Masatoshi Imanishi $\left(\mathbb{D},{ }^{2}, 3\right.$ \\ Yoshiki Toba (D) ${ }^{1,4,5}$ ANd Claudio Ricci (iD 6,7 \\ ${ }^{1}$ Department of Astronomy, Kyoto University, Kitashirakawa-Oiwake-cho, Sakyo-ku, Kyoto 606-8502, Japan \\ ${ }^{2}$ National Astronomical Observatory of Japan, Osawa, Mitaka, Tokyo 181-8588, Japan \\ ${ }^{3}$ Department of Astronomical Science, Graduate University for Advanced Studies (SOKENDAI), 2-21-1 Osawa, Mitaka, Tokyo 181-8588, \\ Japan \\ ${ }^{4}$ Academia Sinica Institute of Astronomy and Astrophysics, $11 \mathrm{~F}$ of Astronomy-Mathematics Building, AS/NTU, No.1, Section 4, \\ Roosevelt Road, Taipei 10617, Taiwan \\ ${ }^{5}$ Research Center for Space and Cosmic Evolution, Ehime University, 2-5 Bunkyo-cho, Matsuyama, Ehime 790-8577, Japan \\ ${ }^{6}$ Núcleo de Astronomía de la Facultad de Ingeniería, Universidad Diego Portales, Av. Ejército Libertador 441, Santiago, Chile \\ ${ }^{7}$ Kavli Institute for Astronomy and Astrophysics, Peking University, Beijing 100871, People's Republic of China
}

(Received January 14, 2020; Revised May 17, 2020; Accepted May 18, 2020)

\begin{abstract}
We have analyzed the broadband X-ray spectra of active galactic nuclei (AGNs) in two "non-merging" luminous infrared galaxies (LIRGs) UGC 2608 and NGC 5135, utilizing the data of NuSTAR, Suzaku, XMM-Newton, and Chandra. Applying the X-ray clumpy-torus model (XCLUMPY: Tanimoto et al. 2019), we find that both sources have similar spectra characterized by Compton-thick (CT) absorption $\left(N_{\mathrm{H}} \sim 5-7 \times 10^{24} \mathrm{~cm}^{-2}\right)$ and small torus angular width $\left(\sigma<20^{\circ}\right)$. The intrinsic $2-10 \mathrm{keV}$ luminosities are $3.9_{-1.7}^{+2.2} \times 10^{43} \mathrm{erg} \mathrm{s}^{-1}$ (UGC 2608) and 2.0 $0_{-1.0}^{+3.3} \times 10^{43} \mathrm{erg} \mathrm{s}^{-1}$ (NGC 5135). The [O IV]-to-nuclear$12 \mu \mathrm{m}$ luminosity ratios are larger than those of typical Seyferts, which are consistent with the torus covering factors $\left(C_{\mathrm{T}} \lesssim 0.7\right)$ estimated from the torus angular widths and column densities by X-ray spectroscopy. The torus covering factors and Eddington ratios $\left(\lambda_{\text {Edd }} \sim 0.1\right)$ follow the relation found by Ricci et al. (2017a) for local AGNs, implying that their tori become geometrically thin due to significant radiation pressure of the AGN that blows out some part of the tori. These results indicate that the CT AGNs in these "non-merger" LIRGs are just a normal AGN population seen edge-on through a large line-of-sight column density. They are in contrast to the buried CT AGNs in late-stage mergers that have large torus covering factors even at large Eddington ratios.
\end{abstract}

Keywords: Black hole physics (159); Active galactic nuclei (16); X-ray active galactic nuclei (2035); Infrared galaxies (790); Supermassive black holes (1663); Observational astronomy (1145)

\section{INTRODUCTION}

Luminous $^{1}$ and ultraluminous ${ }^{2}$ infrared galaxies (U/LIRGs; see Sanders \& Mirabel 1996 for a review) are key populations to understand the co-evolution of galactic bulges and their central supermassive black holes (SMBHs). They are powered by starburst and active

Corresponding author: Satoshi Yamada

styamada@kusastro.kyoto-u.ac.jp

${ }^{1}$ LIRGs: $L_{\mathrm{IR}}(8-1000 \mu \mathrm{m})>10^{11} L_{\odot}=3.828 \times 10^{44} \mathrm{erg} \mathrm{s}^{-1}$

${ }^{2}$ ULIRGs: $L_{\mathrm{IR}}(8-1000 \mu \mathrm{m})>10^{12} L_{\odot}=3.828 \times 10^{45} \mathrm{erg} \mathrm{s}^{-1}$ galactic nucleus (AGN) activities hidden behind gas and dust. The integrated infrared (IR) luminosity of U/LIRGs constitutes a dominant fraction of the total radiation density at $z>1$, which is much larger than that directly visible in the UV band (Madau \& Dickinson 2014). Hence, they trace major processes of star formation and SMBH growth in the cosmic history.

As a possible explanation for the cosmic "downsizing" of galaxies and SMBHs (Cowie et al. 1996; Ueda et al. 2003), it has been suggested that the triggering mechanisms of star formation and mass accretion onto SMBHs may have two channels: more massive galaxies and SMBHs are formed via "major mergers" at higher 
redshifts, while less massive galaxies and SMBHs are former via "secular processes" at lower redshifts (e.g., Kormendy et al. 2011; Draper \& Ballantyne 2012; Alexander \& Hickox 2012; Barro et al. 2013; Tadaki et al. 2014). In fact, morphological studies revealed that LIRGs were generally a mix of mergers and single isolated disk galaxies at $z<1$ (e.g., Wang et al. 2006; Kartaltepe et al. 2010). According to the above scenario, it is expected that the structure of AGNs in "merging" LIRGs and "non-merging" ones may be largely different because of the different AGN triggering mechanisms, even if their IR luminosities (hence star formation rates; SFRs) are similar. Previous studies of "merging" U/LIRGs revealed that at late merger stages the AGNs often became deeply "buried" with large torus covering factors by circumnuclear material of gas and dust (probed in the 1-80 keV broadband X-ray band; e.g., Ricci et al. $2017 \mathrm{~b}$ ) and also of dust (probed in the 1-60 $\mu \mathrm{m}$ near-IR to far-IR band; e.g., Sanders et al. 1988; Imanishi et al. 2008; Yamada et al. 2019). It is thus essential to investigate the properties of AGNs in "non-merging" LIRG utilizing the X-ray and IR data, for a full understanding of whether or not they have distinct characteristics from those in "merging" LIRGs.

In this work, we focus on AGNs in U/LIRGs with no signs of mergers from a complete flux-limited sample of the Great Observatories All-sky LIRG Survey (GOALS; Armus et al. 2009), which consists of 22 ULIRGs and 180 LIRGs having $f_{\nu}(60 \mu \mathrm{m})>5.24 \mathrm{Jy}$ in the local $(z<0.088)$ universe. The GOALS sample contains 12 non-merging LIRGs (according to Stierwalt et al. 2013) that were observed with Nuclear Spectroscopic Telescope Array (NuSTAR; Harrison et al. 2013), the first focusing telescope in orbit operating at $>10 \mathrm{keV}$, by Cycle-4. Among them, NGC 1068 and NGC 7130 are recently reported that they show features of past mergers (Davies et al. 2014; Tanaka et al. 2017), and thus are excluded. We also exclude NGC 1275 (a radio galaxy in the Perseus cluster) and NGC 1365 (showing variable absorption; e.g., Rivers et al. 2015) from our sample as exceptional sources. Performing the same data reduction in Section 2.1, we find that the 6 objects, UGC 2612, NGC 4418, IC 860, NGC 5104, NGC 6907, and NGC 7591 are either undetected or detected only below $\sim 10 \mathrm{keV}$ with NuSTAR. Thus, we select UGC 2608 and NGC 5135 as the best targets for our study.

UGC $2608\left(z=0.0233, \log L_{\mathrm{IR}} / L_{\odot}=11.4\right)$ and NGC $5135\left(z=0.0137, \log L_{\mathrm{IR}} / L_{\odot}=11.3\right)$ are classified as nonmergers from the Spitzer/IRAC $3.6 \mu \mathrm{m}$ and the high resolution Hubble Space Telescope (HST) data (Stierwalt et al. 2013). Optically the sources were clas- sified as Seyfert 2s (Véron-Cetty \& Véron 2003). AGN signatures were also detected at the mid-IR wavelengths on the basis of the $6.2 \mu \mathrm{m}$ polycyclic aromatic hydrocarbon $(\mathrm{PAH})$ equivalent widths and the $[\mathrm{Ne} \mathrm{V}] 14.32 \mu \mathrm{m}$ line luminosities (Inami et al. 2013). The properties of AGNs and starbursts are constrained in the IR band by spectral energy distribution (SED) fitting (e.g., Shangguan et al. 2019).

The nature of the AGNs in UGC 2608 and NGC 5135 are still unclear because of their heavily obscuration. Using XMM-Newton data, Guainazzi et al. (2005) suggested that UGC 2608 contains a heavily obscured AGN with $N_{\mathrm{H}}>1.6 \times 10^{24} \mathrm{~cm}^{-2}$, which is Compton-thick (CT) level (i.e., $N_{\mathrm{H}} \gtrsim 1.5 \times 10^{24} \mathrm{~cm}^{-2}$ ). With Chandra, Levenson et al. (2004) found that the AGN in NGC 5135 is heavily obscured with $N_{\mathrm{H}} \geq 10^{24} \mathrm{~cm}^{-2}$. However, their intrinsic luminosities were not accurately estimated only from the X-ray observations below $10 \mathrm{keV}$. Suzaku detected narrow $\mathrm{Fe} \mathrm{K} \alpha$ lines of large equivalent widths (EWs), $0.6 \pm 0.3 \mathrm{keV}$ for UGC 2608 and $1.6 \pm 0.4 \mathrm{keV}$ for NGC 5135, which are possible evidence for CT AGNs (Fukazawa et al. 2011; Singh et al. 2012), whereas the flux uncertainties above $10 \mathrm{keV}$ are large due to the limited photon statistics. The more sensitive hard X-ray data of NuSTAR observations enable us to characterize the properties of these AGNs.

In this paper, we analyze the best quality broadband X-ray spectra of UGC 2608 and NGC 5135, utilizing the data of NuSTAR, Suzaku, XMM-Newton, and Chandra. The paper is organized as follows. In Section 2, we describe the observations and data reduction. Section 3 reports our results of the spectral analysis using two models: a baseline model and a Monte Carlo-based model from a clumpy torus (XCLUMPY; Tanimoto et al. 2019). We discuss the implications from our results in Section 4. Our findings are summarized in Section 5. Throughout the paper, we adopt the cosmological parameters of $H_{0}=70 \mathrm{~km} \mathrm{~s}^{-1} \mathrm{Mpc}^{-1}, \Omega_{\mathrm{M}}=$ 0.27 , and $\Omega_{\Lambda}=0.73$. All errors correspond to the $90 \%$ confidence level for a single interesting parameter.

\section{OBSERVATIONS AND DATA REDUCTION}

The observation logs of UGC 2608 and NGC 5135 are summarized in Table 1 . The X-ray spectral analysis is carried out by combining the NuSTAR data (Section 2.1) with those of Suzaku (Section 2.2), XMMNewton (Section 2.3), and Chandra (Section 2.4).

\subsection{NuSTAR}

NuSTAR (Harrison et al. 2013) is the first satellite capable of focusing hard X-rays above $10 \mathrm{keV}$, cover- 
Table 1. Observation Log Utilized in This Work

\begin{tabular}{cllclc}
\hline \hline Object & Satellite & \multicolumn{1}{c}{ Instrument } & ObsID & UT Observation Date & Net Exp. (ks) \\
\hline UGC 2608 & NuSTAR & FPMA, FPMB & 60001161002 & 2014 Oct 08 17:06:07 & $22.1 / 22.2$ \\
& Suzaku & XIS-0, 1, 3 & 701007020 & 2007 Feb 04 18:21:52 & 39.5 \\
& XMM-Newton & EPIC/MOS1, 2, pn & 0002942401 & 2002 Jan 28 16:56:46 & $4.8 / 4.8 / 2.1$ \\
NGC 5135 & NuSTAR & FPMA, FPMB & 60001153002 & 2015 Jan 14 15:31:07 & $33.4 / 32.5$ \\
& Suzaku & XIS-0, 1, 3 & 702005010 & 2007 Jul 03 05:59:41 & 52.5 \\
& Chandra & ACIS-S & 2187 & 2001 Sep 04 15:22:37 & 29.3 \\
\hline
\end{tabular}

ing the 3-79 keV band. It consists of two co-aligned Xray telescopes coupled with focal plane modules (FPMA and FPMB; FPMs). NuSTAR observed UGC 2608 for a net exposure of $\sim 22 \mathrm{ks}$ on 2014 October 8 (ObsID = 60001161002 ) and NGC 5135 for $\sim 33$ ks on 2015 January $14($ ObsID $=60001153002)$. The NuSTAR data were processed with the NUSTAR Data Analysis Software NUSTARDAS v1.8.0 included in HEASOFT v6.25, by adopting the calibration files released on 2019 May 13. The NUPIPELINE script was used to produce calibrated and cleaned event files with the SAAMODE $=$ OPTIMIZED and TENTACLE $=$ YES options. The source spectra and light curves were extracted from a circular region with a radius of $50^{\prime \prime}$ by using the NUPRODUCTS task. The background was taken from a nearby source-free circular region with a radius of $100^{\prime \prime}$. We confirmed that the spectra and light curves showed good agreement between FPMA and FPMB, and then we coadded them to improve the photon statistics with the ADDASCASPEC and LCMATH tasks, respectively. The $3-24 \mathrm{keV}$ light curves of the sources show no evidence of significant flux variability on a time scale longer than $5820 \mathrm{~s}$ (the orbital period of $N u S T A R$ ). The spectral bins were grouped to contain at least 50 and 60 counts for UGC 2608 and NGC 5135, respectively, in order to facilitate the use of $\chi^{2}$ statistics and not to lose energy resolution by overbinning.

\subsection{Suzaku}

UGC 2608 and NGC 5135 were observed with Suzaku (Mitsuda et al. 2007) in 2007 February and July, respectively. Suzaku, the fifth Japanese X-ray satellite, carries four X-ray CCD cameras called the X-ray Imaging Spectrometer (XIS-0, XIS-1, XIS-2, and XIS-3), covering the energy band of $0.2-12 \mathrm{keV}$. XIS-0, XIS-2, and XIS-3 are frontside-illuminated cameras (XIS-FIs) and XIS-1 is a backside-illuminated one (XIS-BI). XIS-2 data were not available for both objects due to the malfunction that had occurred in 2006 November. We used the Suzaku calibration database released on 2018 October 23 for generating the cleaned event files with the recommended filtering options. Source photons were extracted from a circular region with a radius of $2.4^{\prime}$ centered on the source center, and the background was from two sourcefree circular regions with radii of $2.2^{\prime}$. We generated the XIS response matrix files (RMF) with XISRMFGEN and ancillary response files (ARF) with XISSIMARFGEN (Ishisaki et al. 2007). All the spectra of the XIS-FIs available in each observation were combined together. The XIS spectra of UGC 2608 and NGC 5135 were rebinned to contain at least 70 and 80 counts per energy bin, respectively. We decided not to use the data of the Hard X-ray Detector (HXD) in our analysis, due to the much poorer photon statistics than those of the $N u S$ $T A R$ data in these faint targets and possible systematic uncertainties in the HXD background model (Fukazawa et al. 2009).

\subsection{XMM-Newton}

The XMM-Newton (Jansen et al. 2001) observation of UGC 2608 was performed on 2002 January 28. We analyzed the data of two EPIC/MOS (MOS1, MOS2) and EPIC/pn reprocessed with the XMM-Newton Science Analysis System (SAS: Gabriel et al. 2004) v17.0.0 and Current Calibration Files (CCF) of 2018 June 22. The raw MOS and pn data files were reduced with the EMPROC and EPPROC tasks, respectively. We selected good events with PATTERN $\leq 12$ for MOS and PATTERN $\leq 4$ for pn. To filter out the periods of background flares, we excluded data when the count rates exceeded 1.0 counts s$^{-1}$ in the $10-12 \mathrm{keV}$ band for MOS and 2.0 counts s $\mathrm{s}^{-1}$ above $10 \mathrm{keV}$ for pn. For both cameras, the source spectra were extracted from a circular region of $20^{\prime \prime}$ radius, whereas the background ones were from a source-free region with a radius of $40^{\prime \prime}$ in the same CCD chips. We created the RMF with RMFGEN and ARF with ARFGEN. The source spectra, background spectra, RMFs, and ARFs of EPIC/MOS1 and MOS2 were combined by using ADDASCASPEC, and the spectral bins were merged to contain no less than 20 counts. 


\subsection{Chandra}

Chandra (Weisskopf et al. 2002) observed NGC 5135 with ACIS-S on 2001 September 04 for a net exposure of $29.3 \mathrm{ks}$. The data reduction was performed with the standard procedures, by using Chandra Interactive Analysis of Observations (CIAO) v4.11 and the Calibration Database (CALDB) v4.8.4.1. The event files were reproduced by the CHANDRA_REPRO tool. We extracted the source spectrum from a circular region with a radius of $10.5^{\prime \prime}$, and took the background from a nearby source-free circular region with the same radius. The spectrum was rebinned to have no less than 20 counts per bin.

\section{X-RAY SPECTRAL ANALYSIS}

The broadband X-ray spectra of these sources $(\sim 0.5-$ $70 \mathrm{keV}$ ) consisting of multiple instrument data are simultaneously analyzed. Considering the signal-to-noise ratio of the spectra, we determine the energy band to be used as follows: for UGC 2608, NuSTAR/FPMs (3$70 \mathrm{keV})$, Suzaku/XIS-FIs (0.9-7 keV), XIS-BI (0.45$7 \mathrm{keV}), X M M-N e w t o n$ EPIC/MOS (0.7-7 keV), and $\mathrm{EPIC} / \mathrm{pn}(0.5-7 \mathrm{keV})$; for NGC 5135, NuSTAR/FPMs (3-74 keV), Suzaku/XIS-FIs (0.8-10 keV), XIS-BI (0.7$8 \mathrm{keV})$, and Chandra/ACIS-S (0.5-6 keV). The data of XIS-FIs and XIS-BI in the 1.6-1.9 keV band are excluded to avoid the calibration uncertainty. ${ }^{3}$ The observed spectra folded with the energy responses are plotted in the left panels of Figure 1 and 2.

In spectral fitting, we consider the Galactic absorption by multiplying phabs to intrinsic spectral models, and fix the hydrogen column densities at $2.03 \times 10^{21} \mathrm{~cm}^{-2}$ for UGC 2608 and $6.02 \times 10^{20} \mathrm{~cm}^{-2}$ for NGC 5135 (Willingale et al. 2013). We correct possible crosscalibration uncertainties among different instruments by introducing constant factors (const1; $C_{\mathrm{FI}}, C_{\mathrm{BI}}, C_{\mathrm{pn}}$, and $C_{\text {ACIS }}$ for XIS-FIs, XIS-BI, EPIC/pn, and ACIS-S, respectively). This value is set to unity for the $N u S$ $T A R / F P M s$ and XMM-Newton EPIC/MOS as calibration references, both of which are well cross-calibrated within a few percent (Madsen et al. 2015, 2017), and allowed the other constants to vary within a range of 0.8-1.2. The solar abundances by Anders \& Grevesse (1989) are assumed. Possible time variability of the AGN emission is ignored because it is not significantly required from the data. All spectral fitting was carried out on XSPEC v12.10.1 (Arnaud 1996) by adopting $\chi^{2}$ statistics.

${ }^{3}$ https://heasarc.gsfc.nasa.gov/docs/suzaku/analysis/abc/node8.html

\subsection{Baseline Model}

Previous X-ray studies suggested that UGC 2608 and NGC 5135 hosted CT AGNs (e.g., Guainazzi et al. 2005; Fukazawa et al. 2011; Singh et al. 2012), and therefore we start with a conventional model often applied for CT objects (e.g., Oda et al. 2017; Ricci et al. 2017c; Tanimoto et al. 2018). The model consists of four components: an absorbed direct component, an unabsorbed scattered component, a reflection component, and a soft thermal component. In the XSPEC terminology, it is described as

$$
\begin{aligned}
& \text { const } 1 * \text { phabs } *(\text { zphabs } * \text { cabs } * \text { zpowerlw } * \text { zhighect } \\
& + \text { const } 2 * \text { zpowerlw } * \text { zhighect }+ \text { pexmon }+ \text { apec }) .
\end{aligned}
$$

The first term represents the transmitted AGN component modeled by an absorbed power law with a highenergy cutoff of $300 \mathrm{keV}$ (e.g., Dadina 2008). We consider the Compton scattering effect to the primary component by multiplying the cabs model. For UGC 2608, the photon index $\left(\Gamma_{\mathrm{AGN}}\right)$ is fixed at 1.8 as a typical value (e.g., Ueda et al. 2014; Ricci et al. 2017c), since it cannot be well constrained due to the limited photon statistics. The second term is the component representing scattering by ionized material. The normalization and cutoff energy are linked to those of the primary component. The photon index $\left(\Gamma_{\text {scat }}\right)$ is set to vary between 1.5 and 3.0, because photoionized plasma often contains emission lines that cannot be resolved by CCD, making the apparent slope steeper than that of the primary component. We multiply the scattering fraction (const2, $f_{\text {scat }}$ ) relative to the transmitted emission at $1 \mathrm{keV}$ (e.g., Ueda et al. 2007). The third term describes the reflection component from the torus. For Compton-reflection continuum we use the pexmon model in XSPEC (Nandra et al. 2007), which calculates a reflection spectrum from optically-thick cold matter (pexrav; Magdziarz \& Zdziarski 1995) including Fe K $\alpha$ (6.4 keV), Fe K $\beta$ (7.1 keV), and Ni K $\alpha(7.5 \mathrm{keV})$ fluorescence lines. The relative intensity, $R=\Omega / 2 \pi(\Omega$ is the solid angle of the reflector), is allowed to vary within a range of $0.1 \leq R \leq 2$ in order to avoid unrealistic solutions, and the inclination angle is set to $i=60^{\circ}$. The normalization, photon index, and cutoff energy are tied to those of the primary component. The last term accounts for optically-thin thermal emission from plasma in the host galaxy (apec; Smith et al. 2001), likely related to star-forming regions.

This analytic model well reproduces the broadband spectra of UGC 2608 (with the chi-squared statistic divided by the number of degrees of freedom, $\chi^{2} /$ dof $=$ $77.0 / 72)$ and NGC $5135\left(\chi^{2} /\right.$ dof $\left.=186.6 / 160\right)$ in the 
Table 2. Summary of the Best-fit Spectral Parameters

\begin{tabular}{|c|c|c|c|c|c|}
\hline \multirow[t]{2}{*}{ No. } & \multirow[t]{2}{*}{ Parameter } & UGC 2608 & \multicolumn{3}{|c|}{ NGC 5135} \\
\hline & & Baseline Model & XCLUMPY Model & Baseline Model & XCLUMPY Model \\
\hline$(1)$ & $N_{\mathrm{H}}^{\mathrm{LOS}}\left[10^{24} \mathrm{~cm}^{-2}\right]$ & $3.2_{-1.0}^{+0.4}$ & $5.4_{-3.1}^{+7.0}$ & $6.3_{-3.1}^{+3.7 a}$ & $6.6_{-2.7}^{+22.5}$ \\
\hline$(2)$ & $N_{\mathrm{H}}^{\mathrm{Equ}}\left[10^{24} \mathrm{~cm}^{-2}\right]$ & $\ldots$ & $15_{-9}^{+19}$ & $\ldots$ & $9.5_{-3.9}^{+32.0}$ \\
\hline (3) & $\Gamma_{\mathrm{AGN}}$ & $1.8^{b}$ & $1.8^{b}$ & $1.50_{-0.00}^{+0.06 a}$ & $1.71_{-0.15}^{+0.19}$ \\
\hline (4) & $A_{\mathrm{AGN}}\left[10^{-2} \mathrm{keV}^{-1} \mathrm{~cm}^{-2} \mathrm{~s}^{-1}\right]$ & $0.65_{-0.42}^{+0.09}$ & $0.96_{-0.41}^{+0.54}$ & $0.59_{-0.49}^{+0.10}$ & $1.27_{-0.64}^{+2.06}$ \\
\hline$(5)$ & $f_{\text {scat }}[\%]$ & $0.87_{-0.26}^{+1.62}$ & $0.57_{-0.24}^{+0.44}$ & $1.84_{-0.28}^{+8.16 a}$ & $0.91_{-0.57}^{+0.77}$ \\
\hline (6) & $\Gamma_{\text {scat }}$ & $3.00_{-0.43}^{+0.00 a}$ & $2.87_{-0.59}^{+0.13 a}$ & $2.79_{-0.10}^{+0.09}$ & $2.65_{-0.11}^{+0.15}$ \\
\hline (7) & $R$ & $0.10_{-0.00}^{+0.18 a}$ & $\ldots$ & $0.10_{-0.00}^{+0.52 a}$ & $\ldots$ \\
\hline (8) & $\sigma[$ degree $]$ & $\ldots$ & $10.0_{-0.0}^{+8.2 a}$ & $\ldots$ & $10.0_{-0.0}^{+4.5 a}$ \\
\hline (9) & $i$ [degree] & $60^{b}$ & $80^{b}$ & $60^{b}$ & $84.0_{-6.0}^{+3.0 a}$ \\
\hline$(10)$ & $N_{\text {Line }}$ & $\ldots$ & $\ldots$ & $\ldots$ & $2.0_{-0.4}^{+0.5}$ \\
\hline$(11)$ & $k \mathrm{~T}[\mathrm{keV}]$ & $0.82_{-0.06}^{+0.13}$ & $0.82_{-0.06}^{+0.14}$ & $0.90_{-0.03}^{+0.03}$ & $0.88_{-0.05}^{+0.03}$ \\
\hline$(12)$ & $A_{\text {apec }}\left[10^{-5} \mathrm{~cm}^{-5}\right]$ & $4.58_{-0.84}^{+1.01}$ & $4.65_{-0.99}^{+1.06}$ & $6.22_{-0.79}^{+0.91}$ & $6.25_{-0.91}^{+1.06}$ \\
\hline (13) & $C_{\mathrm{FI}}$ & $1.20_{-0.14}^{+0.00 a}$ & $1.20_{-0.13}^{+0.00 a}$ & $1.07_{-0.11}^{+0.12}$ & $1.03_{-0.13}^{+0.14}$ \\
\hline (14) & $C_{\mathrm{BI}}$ & $1.20_{-0.11}^{+0.00 a}$ & $1.20_{-0.11}^{+0.00 a}$ & $1.12_{-0.13}^{+0.08 a}$ & $1.08_{-0.14}^{+0.12 a}$ \\
\hline$(15)$ & $C_{\mathrm{pn}}$ & $0.97_{-0.14}^{+0.15}$ & $0.97_{-0.15}^{+0.15}$ & $\ldots$ & $\ldots$ \\
\hline (16) & $C_{\mathrm{ACIS}}$ & $\ldots$ & $\ldots$ & $0.97_{-0.11}^{+0.11}$ & $0.94_{-0.12}^{+0.13}$ \\
\hline$(17)$ & $F_{2-10}^{\mathrm{obs}}\left[10^{-13} \mathrm{erg} \mathrm{s}^{-1} \mathrm{~cm}^{-2}\right]$ & 2.1 & 2.1 & 3.8 & 3.7 \\
\hline (18) & $F_{15-50}^{\text {obs }}\left[10^{-12} \mathrm{erg} \mathrm{s}^{-1} \mathrm{~cm}^{-2}\right]$ & 3.0 & 3.1 & 4.2 & 4.1 \\
\hline (19) & $L_{2-10}\left[10^{43} \mathrm{erg} \mathrm{s}^{-1}\right]$ & $3.2_{-2.0}^{+0.4}$ & $3.9_{-1.7}^{+2.2}$ & $1.6_{-1.3}^{+0.3}$ & $2.0_{-1.0}^{+3.3}$ \\
\hline$(20)$ & $L_{10-50}\left[10^{43} \mathrm{erg} \mathrm{s}^{-1}\right]$ & $3.8_{-2.4}^{+0.5}$ & $5.0_{-2.2}^{+2.8}$ & $3.1_{-2.6}^{+0.5}$ & $3.0_{-1.5}^{+5.0}$ \\
\hline$(21)$ & $L_{\mathrm{K} \alpha}\left[10^{40} \mathrm{erg} \mathrm{s}^{-1}\right]$ & 4.2 & 4.4 & 2.6 & 3.7 \\
\hline$(22)$ & $C_{\mathrm{T}}$ & $\ldots$ & $0.45_{-0.01}^{+0.31 a}$ & $\ldots$ & $0.44_{-0.01}^{+0.20 a}$ \\
\hline \multirow[t]{2}{*}{$(23)$} & $C_{\mathrm{T}}^{(24)}$ & $\cdots$ & $0.28_{-0.03}^{+0.01}$ & $\cdots$ & $0.26_{-0.02}^{+0.01 a}$ \\
\hline & $\chi^{2} /$ dof & $77.0 / 72$ & $75.1 / 72$ & $186.6 / 160$ & $164.1 / 158$ \\
\hline
\end{tabular}

Note-Columns: (1) hydrogen column density along the line of sight; (2) hydrogen column density along the equatorial plane (i.e., the maximum $N_{\mathrm{H}}$ ); (3) power-law photon index of the AGN transmitted component; (4) power-law normalization of the AGN transmitted component at $1 \mathrm{keV}$; (5) scattering fraction; (6) power-law photon index of the scattering component including emission lines from photoionized plasma; (7) reflection strength $(R=\Omega / 2 \pi)$ of the pexmon model; (8) torus angular width; (9) inclination angle of the torus; (10) relative normalization of the emission lines from the torus; (11) temperature of the apec model; (12) normalization of the apec model; (13) cross-calibration of Suzaku/XIS-FIs relative to $N u S T A R / F P M s ; ~(14)$ cross-calibration of Suzaku/XIS-BI relative to $N u S T A R / F P M s$; (15) cross-calibration of XMM-Newton EPIC/pn relative to NuSTAR/FPMs; (16) cross-calibration of Chandra/ACISS relative to NuSTAR/FPMs; (17-18) observed flux in the $2-10 \mathrm{keV}$ and $15-50 \mathrm{keV}$ band, respectively (normalized at the value of the NuSTAR observation); (19-20) intrinsic luminosity in the 2-10 keV and 10-50 keV band, respectively. The errors on luminosities are estimated by fixing the photon index at the best-fit value; (21) luminosity of the iron$\mathrm{K} \alpha$ line; (22-23) torus covering factor derived from the equatorial column density and torus angular width through Equation (4) for Compton-thin $\left(N_{\mathrm{H}} \geq 10^{22} \mathrm{~cm}^{-2}\right)$ and Compton-thick $\left(N_{\mathrm{H}} \geq 10^{24} \mathrm{~cm}^{-2}\right)$ material, respectively.

$a_{\text {The parameter reaches a limit of its allowed range. }}$

${ }^{b}$ Value fixed. 
summarized in Table 2. From the best-fit model, we calculate the observed fluxes, intrinsic AGN luminosities, and $\mathrm{Fe} \mathrm{K} \alpha$ luminosities, which are also listed in Table 2. The line-of-sight column densities are estimated to be $N_{\mathrm{H}}^{\mathrm{LOS}}=3.2_{-1.0}^{+0.4} \times 10^{24} \mathrm{~cm}^{-2}(\mathrm{UGC} 2608)$ and $>3.2$ $\times 10^{24} \mathrm{~cm}^{-2}$ (NGC 5135), classifying both objects as CT AGNs. The folded spectra and best-fit models are plotted in the upper panels of Figure 1 and 2.

\subsection{XCLUMPY Model}

Next, we apply the XCLUMPY model (Tanimoto et al. 2019), a Monte Carlo-based spectral model from the clumpy torus in an AGN. Many observations in the IR band indicate that AGN tori must be composed of dusty clumps rather than of a smooth mixture of gas and dust (e.g., Krolik \& Begelman 1988; Wada \& Norman 2002; Hönig \& Beckert 2007). Moreover, X-ray works using smooth torus models often suggest a large amount of unabsorbed reflection components noticeable below the Fe K edge. These spectral features are interpreted to be caused by clumpy tori (e.g., Liu \& Li 2014; Furui et al. 2016; Tanimoto et al. 2018, 2019). Therefore, we regard it as one of the most physically realistic models currently available.

The biggest advantage of the XCLUMPY model is that the same model can be fitted to the X-ray and the IR data. In this model, the geometry of the torus is assumed to be the same as that in the CLUMPY model in the IR band (Nenkova et al. 2008a,b), where clumps are distributed according to power-law and normal distributions in the radial and angular directions, respectively. In reality, the gas and dust are not located in the same place. ${ }^{4}$ Then, a comparison between self-consistent Xray and IR models provides more robust measures of the covering factors for the "gas" (probed in the X-ray band) and the "dust" (probed in the IR band).

The geometry in the XCLUMPY model is determined by the column density along the equatorial plane $\left(N_{\mathrm{H}}^{\mathrm{Equ}}\right)$, the torus angular width $(\sigma$ within a range of $\left.10^{\circ}-70^{\circ}\right)$, and the inclination angle $(i$ within a range of $\left.20^{\circ}-87^{\circ}\right) .{ }^{5}$ In XSPEC, the model is written as:

$$
\begin{aligned}
& \text { const } 1 * \text { phabs } *(\text { zphabs } * \text { cabs } * \text { zpowerlw } * \text { zhighect } \\
& \text { +const } 2 * \text { zpowerlw } * \text { zhighect }+ \text { atable }\{\text { xclumpy_R.fits }\} \\
& \text { +const } 3 * \text { atable }\{\text { xclumpy_L.fits }\}+\text { apec }) .
\end{aligned}
$$

\footnotetext{
${ }^{4}$ Ichikawa et al. (2019) argued a possible presence of X-ray absorbing dust-free gas. Tanimoto et al. (2020) suggested that the IR spectrum of an AGN is largely affected by a dusty polar outflow.

5 The other fixed parameters are the inner and outer radii of the torus $(0.05 \mathrm{pc}$ and $1.00 \mathrm{pc})$, the radius of each clump (0.002 pc), the number of clumps along the equatorial plane (10.0), and the index of radial density profile (0.5) (see Tanimoto et al. 2019).
}

The first (transmitted component) and second (scattered component by ionized material) terms are the same as in the previous model. The third and fourth ones represent the two table models of XCLUMPY, the reflection continuum (xclumpy_R.fits) and fluorescence lines (xclumpy_L.fits), respectively. The parameters of the reflection continuum and lines are fixed to be the same. The relative normalization of the emission lines to the reflection continuum $\left(N_{\text {Line }}\right.$; const3) is set to be a free parameter for NGC 5135, which significantly improves the fit $\left(\Delta \chi^{2}=30.4\right.$ for 1 dof $)$. This factor can take into account uncertainties caused by the assumption of geometry and possible non-solar abundances. The photon index, cutoff energy, and normalization of the power law are linked to those of the transmitted component. The inclination of UGC 2608 is fixed at $80^{\circ}$, which cannot be well determined with the data. The line-of-sight column density $\left(N_{\mathrm{H}}^{\mathrm{LOS}}\right)$ in the first term is linked to the equatorial column density $\left(N_{\mathrm{H}}^{\mathrm{Equ}}\right)$, the inclination, and the torus angular width through Equation (3) in Tanimoto et al. (2019). The fifth term corresponds to the emission from optically thin thermal plasma in the host galaxy.

We find this model also well reproduces the spectra of UGC $2608\left(\chi^{2} /\right.$ dof $\left.=75.1 / 72\right)$ and NGC $5135\left(\chi^{2} /\right.$ dof $=$ 164.1/158). These fits are statistically better compared with those by the baseline model, although both models represent statistically acceptable representations of the data. Table 2 lists the best-fit parameters, observed fluxes, intrinsic X-ray luminosities, and $\mathrm{K} \alpha$ luminosities. The lower panels of Figure 1 and 2 illustrate the folded spectra and best-fit models. The line-of-sight column densities are found to be $N_{\mathrm{H}}^{\mathrm{LOS}}=5.4_{-3.1}^{+7.0} \times 10^{24} \mathrm{~cm}^{-2}$ and $6.6_{-2.7}^{+22.5} \times 10^{24} \mathrm{~cm}^{-2}$, and the best-fit intrinsic 2$10 \mathrm{keV}$ luminosities are $L_{2-10}=3.9_{-1.7}^{+2.2} \times 10^{43} \mathrm{erg} \mathrm{s}^{-1}$ and $2.0_{-1.0}^{+3.3} \times 10^{43} \mathrm{erg} \mathrm{s}^{-1}$ for UGC 2608 and NGC 5135, respectively. This confirms that both sources host CT AGNs.

\section{DISCUSSION}

We have presented the best quality broadband X-ray spectra of UGC 2608 and NGC 5135 in the $\sim 0.5-70 \mathrm{keV}$ band, observed with NuSTAR, Suzaku, XMM-Newton and Chandra. The sensitive hard X-ray data of $N u S$ $T A R$ have enabled us to best constrain the properties of the obscured AGNs. For both targets, the XCLUMPY model better reproduces the combined spectra than the baseline model, which are found to be dominated by the reflection component from the torus above $10 \mathrm{keV}$. The resultant line-of-sight column densities and intrinsic 2-10 keV luminosities are consistent between the two 

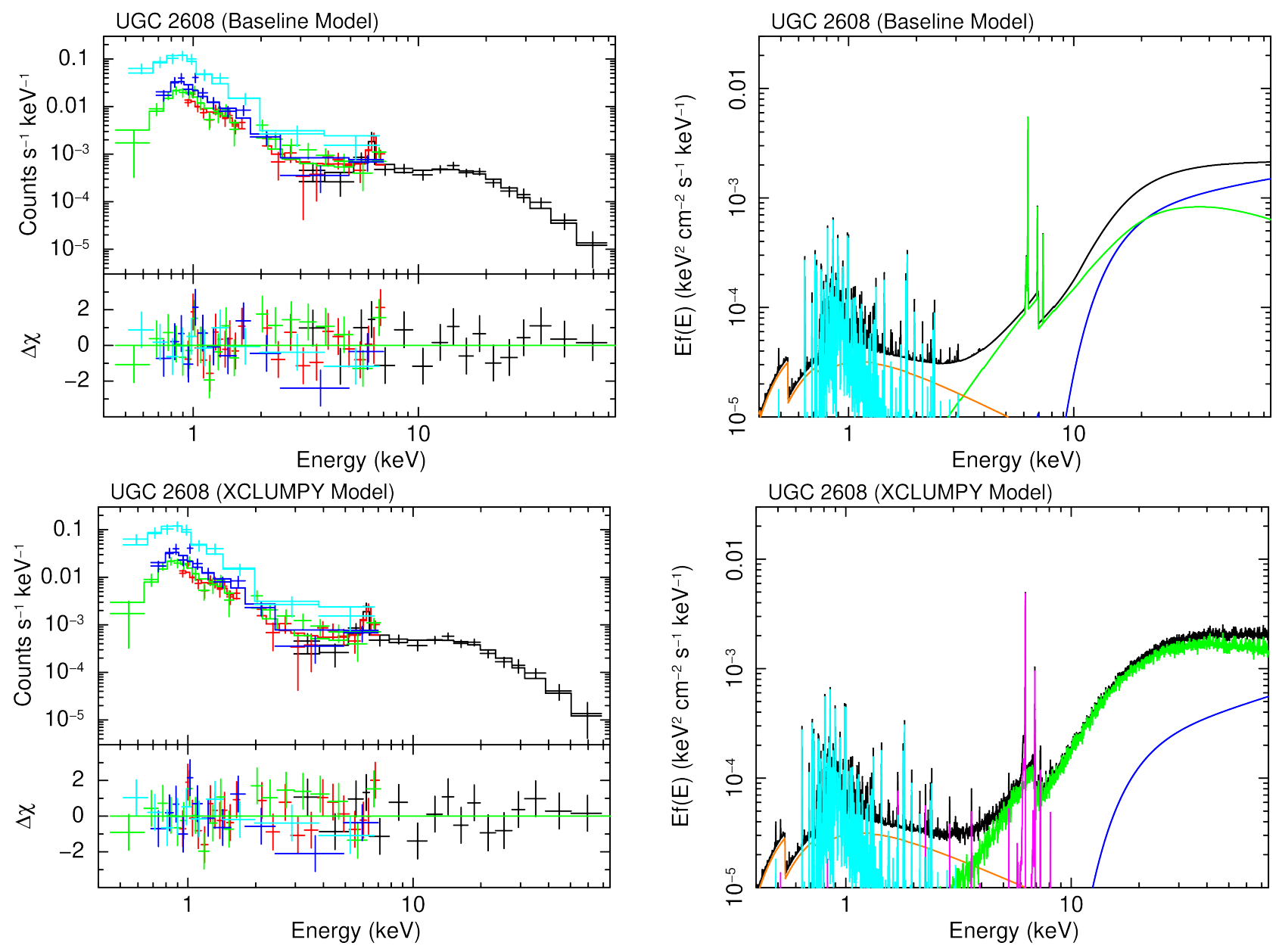

Figure 1. Folded spectra and best-fit models of UGC 2608 with the baseline model (upper panels) and XCLUMPY model (lower panels). Left panels: the black, red, green, blue, and cyan crosses are the data of NuSTAR/FPMs, Suzaku/XIS-FIs, XIS-BI, XMM-Newton EPIC/MOS, and EPIC/pn, respectively. The solid lines represent the best-fit models, and the bottom panels show the residuals. Right panels: the black, blue, orange, green, magenta, cyan lines represent the total, AGN transmitted component, scattering component, reflection component, emission lines from the torus, and thermal emission from the host galaxy, respectively, in units of $E I_{E}$.

models. Hereafter, we refer to the results obtained with the XCLUMPY model, where a more realistic geometry is considered.

We have revealed that UGC 2608 and NGC 5135 contain heavily CT AGNs with column densities of $N_{\mathrm{H}}^{\mathrm{LOS}}$ $=5.4_{-3.1}^{+7.0} \times 10^{24} \mathrm{~cm}^{-2}$ and $6.6_{-2.7}^{+22.5} \times 10^{24} \mathrm{~cm}^{-2}$, respectively. The large Fe K $\alpha$ EWs, $\sim 1.4 \mathrm{keV}$ for UGC 2608 and $\sim 2.5 \mathrm{keV}$ for NGC 5135 (see also Fukazawa et al. 2011), are characteristics of CT AGNs (Ghisellini et al. 1994; Ricci et al. 2014). Our results thus confirm the main conclusions of previous X-ray studies. Using the XMM-Newton spectra of UGC 2608, Guainazzi et al. (2005) obtained a photon index of $\Gamma=2.3_{-0.3}^{+1.1}$, which is assumed to be common for the AGN and scattered components, and a hydrogen column density of $N_{\mathrm{H}}>1.6 \times 10^{24} \mathrm{~cm}^{-2}$. Analyzing the Suzaku 0.5$50 \mathrm{keV}$ spectra of NGC 5135, Singh et al. (2012) ob- tained $\Gamma_{\text {AGN }}=1.65_{-0.12}^{+0.26}$ and $\Gamma_{\text {scat }}=2.31_{-0.07}^{+0.12}$ with a column density of $N_{\mathrm{H}}=2.20_{-0.27}^{+0.36} \times 10^{24} \mathrm{~cm}^{-2}$. This column density of NGC 5135 is smaller by a factor of $\sim 3$ than our result. We infer that the difference may be due to a calibration issue and/or time variability in $N_{\mathrm{H}}$, since the Suzaku HXD/PIN flux in the $15-50 \mathrm{keV}$ band $^{6}, F_{15-50}^{\text {obs }}=(1.09 \pm 0.13) \times 10^{-11} \mathrm{erg} \mathrm{cm}^{-2} \mathrm{~s}^{-1}$, was $\sim 3$ times larger than the best-fit NuSTAR flux.

\subsection{Luminosities and Eddington Ratios}

In the IR band, the presence of the obscured AGNs in UGC 2608 and NGC 5135 was suggested by detections

\footnotetext{
${ }^{6}$ The error includes a systematic uncertainty of $\lesssim 3 \%$ in the HXD/PIN background model (Fukazawa et al. 2009). The bestfit photon index of 1.71 is assumed to convert from the observed count rate into the $15-50 \mathrm{keV}$ flux.
} 

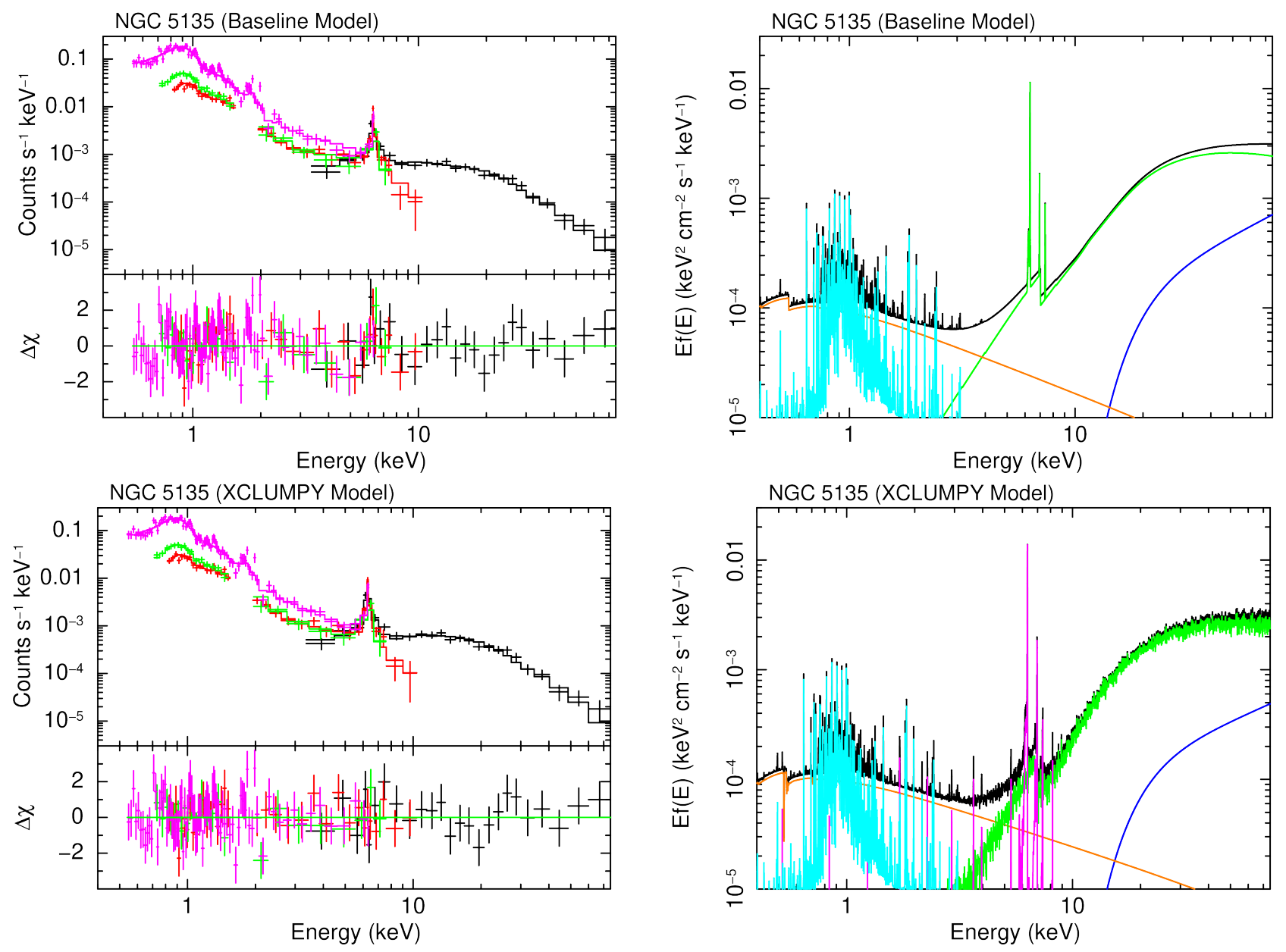

Figure 2. Folded spectra and best-fit models of NGC 5135 with the baseline model (upper panels) and XCLUMPY model (lower panels). Left panels: the black, red, green, and magenta crosses are the data of NuSTAR/FPMs, Suzaku/XIS-FIs, XIS-BI, and Chandra/ASIS-S, respectively. The solid lines represent the best-fit models, and the bottom panels show the residuals. Right panels: the black, blue, orange, green, magenta, cyan lines represent the total, AGN transmitted component, scattering component, reflection component, emission lines from the torus, and thermal emission from the host galaxy, respectively, in units of $E I_{E}$.

of high-excitation emission lines, in particular of [O IV] $25.89 \mu \mathrm{m}$ and [Ne V] $14.32 \mu \mathrm{m}$ (Inami et al. 2013); using the five diagnostics of Spitzer/IRS data ${ }^{7}$, Díaz-Santos et al. (2017) estimated the AGN fractions in the mid-IR luminosities to be $\sim 62 \%$ for UGC 2608 and $\sim 36 \%$ for NGC 5135 (the average of the five methods). Spectral decomposition can constrain the physical properties of the AGN in more detail. From the broadband IR $(\sim 1-$ $500 \mu \mathrm{m}$ ) SED fitting, Shangguan et al. (2019) estimated the stellar masses, $\log \left(M_{\text {stellar }} / M_{\odot}\right)=10.92 \pm 0.20$ and $11.03 \pm 0.20$, the SFRs, $\log \left(\mathrm{SFR} / M_{\odot} \mathrm{yr}^{-1}\right)=1.37 \pm 0.20$

\footnotetext{
7 The diagnostics are following: the $[\mathrm{Ne} \mathrm{V}]_{14.3} /[\mathrm{Ne} \mathrm{II}]_{12.8}$ flux ratios; the $[\mathrm{O} \mathrm{IV}]_{25.9} /[\mathrm{Ne} \mathrm{II}]_{12.8}$ flux ratios; the equivalent width of the $6.2 \mu \mathrm{m} \mathrm{PAH}$; the $S_{30} / S_{15}$ dust continuum slope; and the Laurent diagram (Laurent et al. 2000).
}

and $1.34 \pm 0.01$, and the IR luminosities from the tori, $\log \left(L_{\text {torus }}^{\text {(IR) }} / \mathrm{erg} \mathrm{s}^{-1}\right)=44.18_{-0.04}^{+0.05}$ and $43.76_{-0.03}^{+0.03}$ for UGC 2608 and NGC 5135, respectively. The intrinsic 2-10 keV luminosity of a normal Seyfert galaxy can be estimated from an IR AGN luminosity by using the relation by Mullaney et al. (2011):

$$
\log L_{2-10,43}=\frac{\log L_{\mathrm{IR}, 43}^{\mathrm{AGN}}-(0.53 \pm 0.26)}{(1.11 \pm 0.07)}
$$

where the X-ray and IR luminosities are in units of $10^{43} \mathrm{erg} \mathrm{s}^{-1}$. Assuming $L_{\text {torus }}^{(\mathrm{IR})} \approx L_{\mathrm{IR}}^{\mathrm{AGN}}$, the above equation yields X-ray luminosities of $L_{2-10}=(3.9 \pm 2.6) \times$ $10^{43} \mathrm{erg} \mathrm{s}^{-1}$ (UGC 2608) and $(1.6 \pm 1.1) \times 10^{43} \mathrm{erg} \mathrm{s}^{-1}$ (NGC 5135). They are in good agreement with our results derived from the broadband X-ray spectral analysis. 
Table 3. Nuclear $12 \mu \mathrm{m}$ and Intrinsic $2-10 \mathrm{keV}$ Luminosity for AGNs in the Local U/LIRGs

\begin{tabular}{lcccc}
\hline \hline Object & M & $\log L_{12 \mu \mathrm{m}}^{\text {(nuc) }}$ & $\log L_{2-10}$ & Ref \\
$(1)$ & $(2)$ & $(3)$ & $(4)$ & $(5)$ \\
\hline NGC 34 & $\mathrm{D}$ & $43.08 \pm 0.05$ & $41.98 \pm 0.60$ & 1 \\
NGC 235A & $\mathrm{B}$ & $43.31 \pm 0.20$ & $43.18 \pm 0.30$ & 1 \\
NGC 1068 & $\mathrm{N}$ & $43.80 \pm 0.15$ & $43.64 \pm 0.30$ & 1 \\
NGC 1365 & $\mathrm{N}$ & $42.54 \pm 0.04$ & $42.12 \pm 0.20$ & 1 \\
IRAS 05189-2524 & $\mathrm{D}$ & $44.87 \pm 0.17$ & $43.73 \pm 0.43$ & 1 \\
UGC 5101 & $\mathrm{D}$ & $44.35 \pm 0.08$ & $43.15 \pm 0.40^{a}$ & 2 \\
NGC 3690W & $\mathrm{C}$ & $43.73 \pm 0.28$ & $43.26 \pm 0.60$ & 1 \\
NGC 3690E & $\mathrm{C}$ & $43.30 \pm 0.24$ & $39.76 \pm 0.30$ & 1 \\
IC 883 & $\mathrm{D}$ & $<43.90$ & $40.99 \pm 0.40^{a}$ & 3 \\
MCG-03-34-064 & $\mathrm{A}$ & $44.00 \pm 0.05$ & $43.33 \pm 0.48$ & 1 \\
IC 4518W & $\mathrm{B}$ & $43.54 \pm 0.07$ & $43.05 \pm 0.59$ & 1 \\
ESO 286-19 & $\mathrm{D}$ & $44.67 \pm 0.04$ & $42.39 \pm 0.30$ & 1 \\
NGC 7130 & $\mathrm{N}$ & $43.17 \pm 0.09$ & $42.81 \pm 0.60$ & 1 \\
NGC 7469 & $\mathrm{A}$ & $43.83 \pm 0.05$ & $43.19 \pm 0.07$ & 1 \\
NGC 7674 & $\mathrm{A}$ & $44.26 \pm 0.06$ & $44.02 \pm 0.55$ & 1 \\
NGC 7679 & $\mathrm{A}$ & $42.74 \pm 0.12$ & $42.03 \pm 0.67$ & 1 \\
\hline UGC 2608 & $\mathrm{N}$ & $43.63(<43.84)^{b}$ & $43.56 \pm 0.22$ & 4 \\
NGC 5135 & $\mathrm{N}$ & $43.24 \pm 0.08$ & $43.26 \pm 0.28$ & 4 \\
\hline
\end{tabular}

Note-Columns: (1) Object name; (2) merger stage ( $\mathrm{N}=$ nonmerger, $\mathrm{A}=$ pre-merger, $\mathrm{B}=$ early-stage merger, $\mathrm{C}=$ mid-stage merger, and D = late-stage merger; see Yamada et al. 2019); (3) nuclear $12 \mu \mathrm{m}$ luminosity in erg s${ }^{-1}$; (4) intrinsic $2-10 \mathrm{keV}$ luminosity in $\mathrm{erg} \mathrm{s}^{-1}$; (5) references for the intrinsic 2-10 keV luminosity. List of references: (1) Asmus et al. (2015); (2) Oda et al. (2017); (3) Romero-Cañizales et al. (2017); (4) this work.

$a_{\text {Typical error is adopted. }}$

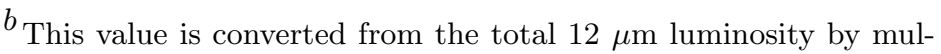
tiplying the mid-IR AGN fraction.

The BH masses $\left(M_{\mathrm{BH}}\right)$ of UGC 2608 and NGC 5135 are estimated as $\log \left(M_{\mathrm{BH}} / M_{\odot}\right) \sim 7.78 \pm 0.50$ (Dasyra et al. 2011) and $\sim 7.29 \pm 0.44$ (Marinucci et al. 2012), respectively, on the basis of the $M_{\mathrm{BH}^{-}} \sigma$ relation. ${ }^{8}$ Adopting a $2-10 \mathrm{keV}$-to-bolometric luminosity ratio of 20 , a typical value for Seyferts (Vasudevan \& Fabian 2007), we can convert our best-fit X-ray luminosities to the bolometric ones as $L_{\mathrm{bol}}^{\mathrm{AGN}}=7.8_{-3.3}^{+4.4} \times 10^{44} \mathrm{erg} \mathrm{s}^{-1}$ (UGC 2608) and $4.1_{-2.1}^{+6.6} \times 10^{44} \mathrm{erg} \mathrm{s}^{-1}$ (NGC 5135).

8 These BH masses are consistent with those calculated from the stellar masses of the host galaxies by using the empirical relation of Reines \& Volonteri (2015).

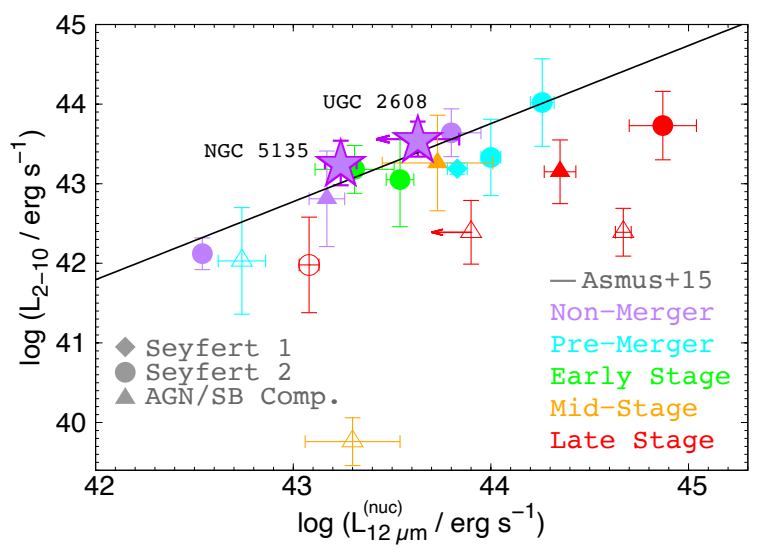

Figure 3. Nuclear $12 \mu \mathrm{m}$ luminosity vs. intrinsic $2-10 \mathrm{keV}$ luminosity for the local U/LIRGs in Table 3. These symbols are color coded by the merger stages determined by Stierwalt et al. (2013). Diamonds, circles, and triangles mark the Seyfert 1/1.5, Seyfert 1.8/2, and AGN/starburst composites determined by the optical AGN classification, and empty symbols are the starburst-dominated objects whose mid-IR AGN fractions are $<1 / 3$ (see Yamada et al. 2019). Purple stars represent UGC 2608 and NGC 5135. Arrows mark upper limits, and black solid line shows the typical relation for local AGNs (Asmus et al. 2015).

Then, the Eddington ratios are calculated to be $\lambda_{\text {Edd }}=$ $0.10_{-0.07}^{+0.25}$ (UGC 2608) and $0.17_{-0.12}^{+0.51}$ (NGC 5135).

These results $\left(\lambda_{\mathrm{Edd}} \sim 0.1\right)$ are in contrast to the AGNs in late-stage merging U/LIRGs, which often have large Eddington ratios of $\gtrsim 0.5$, such as Mrk 463E $\left(\lambda_{\mathrm{Edd}} \sim\right.$ 0.4-0.8; Yamada et al. 2018), IRAS 05189-2524 ( $\lambda_{\text {Edd }} \sim$ 1.2; Teng et al. 2015), and Mrk 231 ( $\lambda_{\text {Edd }} \sim 5.2$; Teng et al. 2015). Thus, the SMBHs in these "non-merging" U/LIRGs are not so rapidly growing as those in typical "merging" U/LIRGs.

\subsection{Geometry of Narrow Line Region and Torus}

The comparison between intrinsic X-ray and nuclear (subarcsecond-scale) $12 \mu \mathrm{m}$ luminosities is useful to investigate the environment in the central regions. $\mathrm{Nu}-$ clear $12 \mu \mathrm{m}$ luminosity originates mainly from hot dust heated by an AGN (e.g., Gandhi et al. 2009), which are obtained from high spatial-resolution observations in order to minimize the contamination by the host galaxy (Asmus et al. 2014). In fact, Asmus et al. (2015) found that the nuclear $12 \mu \mathrm{m}$ and intrinsic X-ray luminosities were well correlated for normal Seyferts, whereas they were not for AGN/starburst composites, uncertain AGNs (e.g., low-luminosity AGNs), and buried AGNs with large torus covering factors (Yamada et al. 2019).

The nuclear $12 \mu \mathrm{m}$ luminosities are obtained by Asmus et al. (2014) for $23 \mathrm{U} /$ LIRGs in the GOALS sample. In Table 3, we select AGNs in various merging stages of 
these U/LIRGs (Yamada et al. 2019) whose intrinsic 2$10 \mathrm{keV}$ luminosities are estimated. Figure 3 shows the relation between X-ray and nuclear $12 \mu \mathrm{m}$ luminosities. As shown in the figure, in the case of mid-/late-stage mergers, the X-ray to nuclear $12 \mu \mathrm{m}$ luminosity ratios are smaller than the typical relation found for normal Seyferts (Asmus et al. 2015). There are two possibilities that (1) the X-ray-to-bolometric luminosity ratio is small because of high Eddington ratios (Vasudevan \& Fabian 2007) and that (2) starburst contamination to the $12 \mu \mathrm{m}$ luminosity is significant even in the nuclear region. Whereas, UGC $2608^{9}$ and NGC 5135 show the ratios consistent with the typical relation found for normal Seyferts, indicating that they are neither AGNs with large Eddington ratios nor starburst-dominant objects in agreement with their SFRs.

The properties of the tori in non-merging LIRGs are still unclear. The IRAS $25-60 \mu \mathrm{m}$ flux ratios $\left(f_{25} / f_{60}\right)$ of 0.43 for UGC 2608 and 0.34 for NGC 5135 (Sanders et al. 2003) show warm far-IR colors $(>0.2)$, supporting that they are not buried AGNs but normal edge-on CT AGNs with co-existing nuclear starbursts (Sanders et al. 1988; Imanishi et al. 2006, 2007). To confirm the torus geometry, the [O IV] $25.89 \mu \mathrm{m}$ and X-ray luminosity ratio provides the information of the spatial extent of the narrow line region (NLR). The [O IV] line is emitted from the NLR irradiated by UV light from an AGN. Hence, its luminosity should be roughly proportional to the AGN bolometric luminosity times the solid angle of the NLR (e.g., Kawamuro et al. 2016). However, this relation is also affected by the ratio of the bolometric luminosity to the X-ray one; for instance, AGNs with high Eddington ratio such as those in the late-stage merging U/LIRGs may have large ratios (i.e., X-ray weak; e.g., Teng et al. 2015; Yamada et al. 2018; Toba et al. 2019).

To avoid the uncertainty by this effect, we investigate the correlation between the [O IV] $25.89 \mu \mathrm{m}$ line and nuclear $12 \mu \mathrm{m}$ continuum luminosities. This would be a better tracer of covering factors because both are determined mainly by the UV luminosity dominating the total power of the intrinsic AGN radiation (Yamada et al. 2019). The sample is selected from our targets and the local U/LIRGs listed in Table 2 of Yamada et al. (2019), whose [O IV] and nuclear $12 \mu \mathrm{m}$ luminosities are both available. The result is shown in Figure 4. They reported that the AGNs in the mid- to late-stage

${ }^{9}$ For UGC 2608 , the "nuclear" $12 \mu \mathrm{m}$ luminosity is not available. Hence, we utilize the total $12 \mu \mathrm{m}$ luminosity $\left[(6.97 \pm 0.04) \times 10^{43}\right.$ $\mathrm{erg} \mathrm{s}^{-1}$ obtained from the Spitzer/IRS spectra as a maximum value] by multiplying the mid-IR AGN fraction ( $62 \%$; DíazSantos et al. 2017).

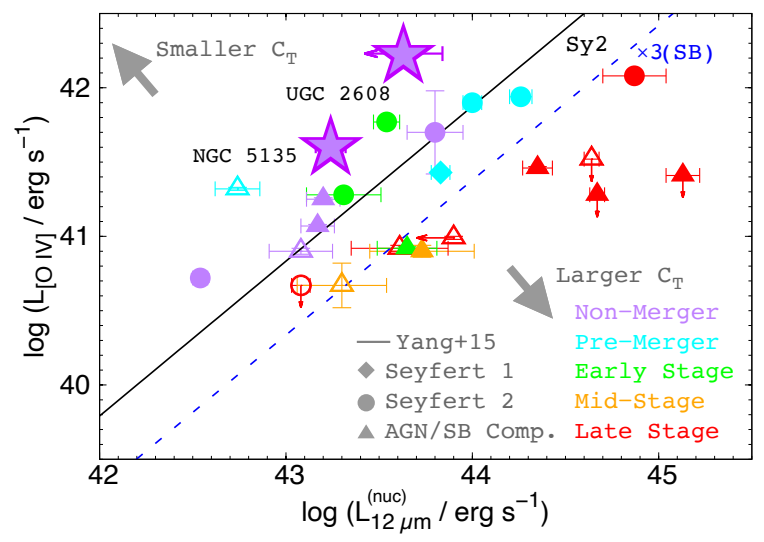

Figure 4. Relation of the [O IV] $25.89 \mu \mathrm{m}$ to nuclear $12 \mu \mathrm{m}$ luminosity for our targets and the AGNs in the local U/LIRGs (Yamada et al. 2019). Symbols are the same as in Figure 3. The black solid and blue dashed lines are the averaged relation for Seyfert 2 s obtained by Yang et al. (2015) and that corrected for contribution of starburst in the mid-IR luminosity by a factor of 3 , which is valid for objects with mid-IR AGN fractions of 1/3 (a typical value in starburst-dominant objects).

mergers of U/LIRGs have small [O IV] luminosities relative to the $12 \mu \mathrm{m}$ ones, suggesting that their nuclei are deeply buried by tori with large covering factors. By contrast, the AGNs in UGC 2608 and NGC 5135 have larger [O IV] luminosities than those of typical Seyfert $2 \mathrm{~s}$ (Yang et al. 2015) and the other U/LIRGs, indicating that the solid angles of their NLRs are large (i.e., the torus covering factors are small).

\subsection{Covering factors in the $X$-ray and IR band}

To reinforce our interpretation on the torus geometry of UGC 2608 and NGC 5135, we estimate the covering factor $\left(C_{\mathrm{T}}\right)$ from our X-ray spectral analysis results. With the XCLUMPY model, we have determined the torus angular widths to be $\sigma<18.2^{\circ}$ degrees and $<14.5^{\circ}$, and the equatorial hydrogen column densities to be $N_{\mathrm{H}}^{\mathrm{Equ}}=15_{-9}^{+19} \times 10^{24} \mathrm{~cm}^{-2}$ and $9.5_{-3.9}^{+32.0} \times 10^{24} \mathrm{~cm}^{-2}$ for UGC 2608 and NGC 5135, respectively. These torus angular widths are smaller than the averaged value of the 10 local Seyfert $2 \mathrm{~s}\left(\langle\sigma\rangle \sim 30^{\circ}\right)$ analyzed by Tanimoto et al. (2020) with XCLUMPY. Figure 5 shows the confidence contours between $N_{\mathrm{H}}^{\mathrm{Equ}}$ and $\sigma$. In the geometry of XCLUMPY, the mean column density at a given elevation angle $\left(\theta \equiv 90^{\circ}-i\right)$ can be calculated as

$$
N_{\mathrm{H}}(\theta)=N_{\mathrm{H}}^{\mathrm{Equ}} \exp \left[-\left(\frac{\theta}{\sigma}\right)^{2}\right] \text {. }
$$

Defining $\theta_{\mathrm{c}}$ as the angle for which $N_{\mathrm{H}}=10^{22} \mathrm{~cm}^{-2}$, we find $\theta_{\mathrm{c}}<49.4^{\circ}$ and $<39.6^{\circ}$, which correspond to 

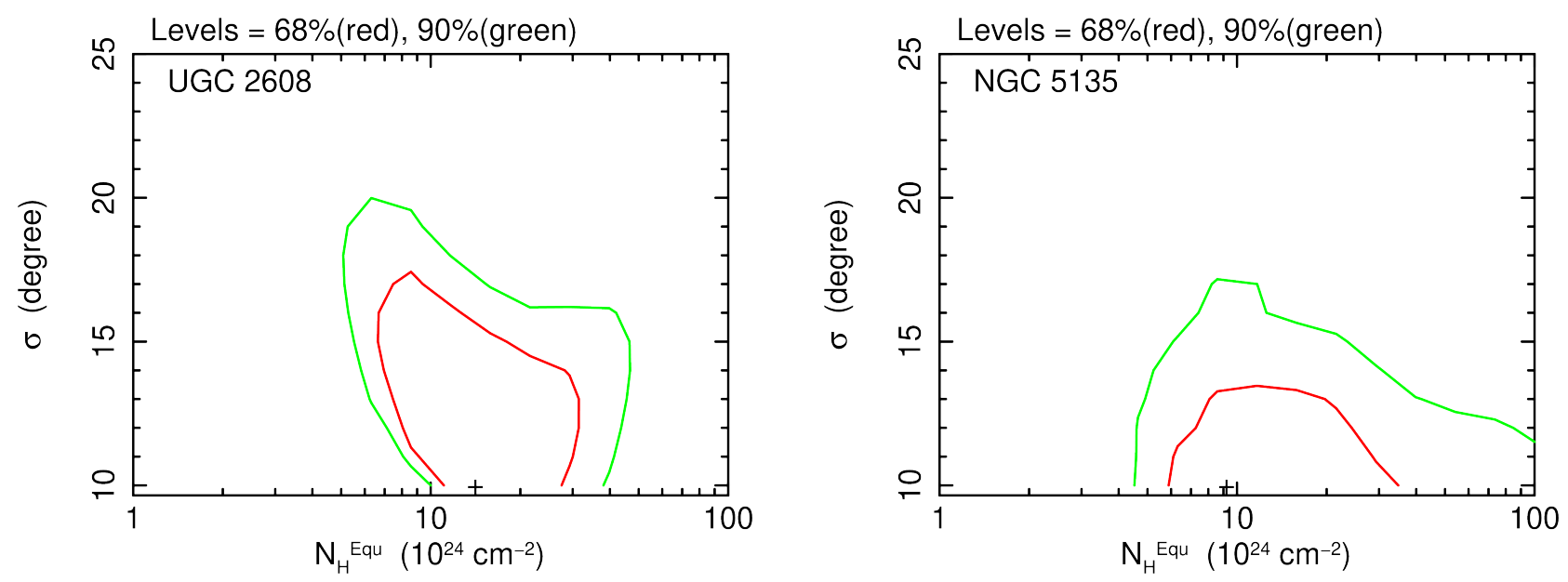

Figure 5. Confidence contours between $N_{\mathrm{H}}^{\text {Equ }}$ and $\sigma$ of UGC 2608 (left panel) and NGC 5135 (right panel). The red and green lines show the confidence levels at $1 \sigma$ (red) and $90 \%$ (green), respectively.

$C_{\mathrm{T}}<0.76$ and $<0.64$ for UGC 2608 and NGC 5135, respectively. These results imply that these AGNs are not deeply buried.

Considering the mid-IR results in Section 4.2, the AGNs in UGC 2608 and NGC 5135 have small covering factors for both gas and dust. Ichikawa et al. (2015) analyzed the IR SED of NGC 5135 with the CLUMPY model and obtained a torus angular width of $\sigma_{\mathrm{IR}}=63_{-5}^{+3}$ degrees and a covering factor of $C_{\mathrm{T}, \mathrm{IR}}=0.97_{-0.04}^{+0.01}$ (the errors are $1 \sigma$ ). These estimates are larger than those of X-ray results $\left(\sigma<14.5^{\circ}\right.$ and $\left.C_{\mathrm{T}}<0.76\right)$. Similar trends are reported in case of local Seyfert $1 \mathrm{~s}$ (Ogawa et al. 2019) and Seyfert 2s (Tanimoto et al. 2019, 2020) by comparing the results with the XCLUMPY and CLUMPY models. Tanimoto et al. (2020) and Ogawa et al. (2020) find a significant tendency that $\sigma_{\mathrm{IR}}$ estimated by Ichikawa et al. (2015) and García-Bernete et al. (2019) is larger than $\sigma_{\mathbf{X}}$, particularly in a high inclination system. They propose that the discrepancy can be explained by a contribution from "dusty" polar outflows in the IR flux (Tristram et al. 2014; Asmus et al. 2016; Asmus 2019). These effects are not considered in the CLUMPY model, whereas the effects to the X-ray spectra are expected to be small (Tanimoto et al. 2020). Thus, the results from the X-ray spectra with XCLUMPY give more realistic estimates of the torus covering factors than those from the IR SED with CLUMPY.

Even if the nuclear $12 \mu \mathrm{m}$ luminosity ratio may contain the emission from the polar outflows, the [O IV] to nuclear $12 \mu \mathrm{m}$ luminosity ratio still represents the spatial extent of the NLR. Hence, we conclude that AGN tori in UGC 2608 and NGC 5135 are geometrically thin, on the basis of the X-ray spectra and the [O IV] to nuclear $12 \mu \mathrm{m}$ luminosity ratios.

\subsection{Covering Factors vs. Eddington ratios}

We investigate the relation between the covering factor and Eddington ratio. Utilizing local AGNs in the Swift/BAT 70-month catalog, Ricci et al. (2017a) report that AGNs with $10^{-4} \leq \lambda_{\text {Edd }} \leq 10^{-1.5}$ have obscurers of $N_{\mathrm{H}} \geq 10^{22} \mathrm{~cm}^{-2}$ with large covering factors $\left(C_{\mathrm{T}} \sim 0.85\right)$, whereas AGNs with $\lambda_{\mathrm{Edd}} \geq 10^{-1.5}$ have those with smaller covering factors $\left(C_{\mathrm{T}} \sim 0.40\right)$. They interpret that radiation pressure from the AGN expells dusty material when $\lambda_{\text {Edd }} \geq 10^{-1.5}$.

By adopting the estimated Eddington ratios (Section 4.1), the relation of Ricci et al. (2017a) predicts the covering factors with $N_{\mathrm{H}} \geq 10^{22} \mathrm{~cm}^{-2}$ to be $C_{\mathrm{T}}=$ 0.32-0.76 (UGC 2608) and 0.31-0.72 (NGC 5135), and that with $N_{\mathrm{H}} \geq 10^{24} \mathrm{~cm}^{-2}$ to be $C_{\mathrm{T}}^{(24)} \sim 0.22$ (both objects). ${ }^{10}$ These values match well with the obtained covering factors in the XCLUMPY model $\left(C_{\mathrm{T}} \sim 0.45\right.$ and $\sim 0.44$, and $C_{\mathrm{T}}^{(24)} \sim 0.28$ and $\sim 0.26$ for $\mathrm{UGC} 2608$ and NGC 5135, respectively). The results suggest that the XCLUMPY model reproduces a realistic description of their compact AGN tori with $N_{\mathrm{H}} \sim 10^{24} \mathrm{~cm}^{-2}$ obscuration; foreground absorption by the host galaxy (typically $N_{\mathrm{H}} \sim 10^{22} \mathrm{~cm}^{-2}$ ), if any, is negligible. We infer that their tori become geometrically thin due to significant radiation pressure of the AGN as is the case of local AGNs with moderately high Eddington ratios.

Thus, our results indicate that the CT AGNs in these "non-merging" LIRGs are just a normal AGN population with moderate Eddington ratios seen close to edgeon through a large line-of-sight column density. We note

\footnotetext{
10 The $1 \sigma$ uncertainties are \pm 0.08 (UGC 2608) and \pm 0.09 (NGC 5135) for $C_{\mathrm{T}}$, and \pm 0.07 (both) for $C_{\mathrm{T}}^{(24)}$.
} 
that these two objects may have smaller covering factors than typical values in non-merging LIRGs because they have largest $[\mathrm{O}$ IV]-to-12 $\mu \mathrm{m}$ luminosity ratios among the 24 local U/LIRGs in Figure 4. This may be due to a selection bias by the detections with $N u S T A R$ for AGNs with high luminosities and hence with large Eddington ratios. It is thus important to confirm if all non-merging LIRGs follow the relation by (Ricci et al. 2017a), using a larger sample by more sensitive broadband X-ray spectroscopy.

By contrast, many studies suggest that AGNs in U/LIRGs of late-stage mergers are deeply buried (i.e., having tori with large covering factors reaching almost unity; Imanishi et al. 2006, 2007; Ricci et al. 2017b; Yamada et al. 2019) even if they shine at large Eddington ratios (Teng et al. 2015; Oda et al. 2017; Yamada et al. 2018). Then, the driver of these phenomena would be not the Eddington ratio but the merger environment. In such a "merging" system, the column density in almost all directions becomes too large to be blowed out by radiation pressure of the AGN. Thus, we suggest that AGNs in "non-merging" LIRGs and those in "merging" ones are distinct populations. The former have small to moderate Eddington ratios and are not buried, while the latter have moderate to high Eddington ratios even though they are deeply "buried". This is probably because the AGN trigger mechanism in "non-merging" LIRGs is less violent than major mergers, such as minor mergers, fly-by companions, and/or secular evolution (e.g., Alonso-Herrero et al. 2013).

\section{SUMMARY AND CONCLUSIONS}

In this paper, we study the best-quality broadband $\mathrm{X}$ ray spectra of the two "non-merging" LIRGs UGC 2608 and NGC 5135, by combining the data of $N u S T A R$, Suzaku, XMM-Newton, and Chandra. Applying the XCLUMPY model (Tanimoto et al. 2019), we estimate the line-of-sight hydrogen column densities of $N_{\mathrm{H}}^{\mathrm{LOS}}=$ $5.4_{-3.1}^{+7.0} \times 10^{24} \mathrm{~cm}^{-2}$ and $6.6_{-2.7}^{+22.5} \times 10^{24} \mathrm{~cm}^{-2}$, and the intrinsic $2-10 \mathrm{keV}$ luminosities to be $L_{2-10}=3.9_{-1.7}^{+2.2}$ $\times 10^{43} \mathrm{erg} \mathrm{s}^{-1}$ and $2.0_{-1.0}^{+3.3} \times 10^{43} \mathrm{erg} \mathrm{s}^{-1}$ for UGC 2608 and NGC 5135, respectively. Thus, both objects are firmly classified as CT AGNs.

The Eddington ratios of both targets are estimated to be $\sim 0.1$, by using the black hole masses (Dasyra et al. 2011; Marinucci et al. 2012) and the intrinsic 2$10 \mathrm{keV}$ luminosities obtained from our careful analysis. From the X-ray spectra, we determine the torus angular widths of UGC 2608 and NGC 5135 to be $\sigma<20^{\circ}$, and the covering factors of material with $N_{\mathrm{H}}>10^{22} \mathrm{~cm}^{-2}$ to be $C_{\mathrm{T}} \lesssim 0.7$. The covering factors and Eddington ratios are consistent with the relation found for local Swift/BAT AGNs by Ricci et al. (2017a), implying that their tori are geometrically thin because of radiation pressure of the AGN to dusty material.

Our results indicate that the CT AGNs in these "nonmerging" LIRGs are just a normal AGN population with moderate Eddington ratios seen close to edgeon through a large line-of-sight column density. By contrast, AGNs in U/LIRGs of late-stage mergers are deeply buried (Imanishi et al. 2006; Ishisaki et al. 2007; Ricci et al. 2017b; Yamada et al. 2019) despite of their large Eddington ratios (Teng et al. 2015; Oda et al. 2017; Yamada et al. 2018). This suggests that AGNs in "nonmerging" LIRGs and those in "merging" ones are distinct populations.

\section{ACKNOWLEDGMENTS}

We thank the anonymous referee for helpful suggestions, which helped us improve the original manuscript. This research has made use of the NuSTAR Data Analysis Software (NuSTARDAS) jointly developed by the ASI Science Data Center (ASDC, Italy) and the California Institute of Technology (Caltech, USA). This work makes use of data obtained from the Suzaku satellite, a collaborative mission between the space agencies of Japan (JAXA) and the USA (NASA). This publication makes use of data obtained with $X M M$-Newton, an ESA science mission with instruments and contributions directly funded by ESA Member States and NASA, and with Chandra, supported by the Chandra X-ray Observatory Center, which is operated by the Smithsonian Astrophysical Observatory for and on behalf of NASA. This publication also makes use of the data from the NASA/IPAC Infrared Science Archive and NASA/IPAC Extragalactic Database (NED), which are operated by the Jet Propulsion Laboratory, California Institute of Technology, under contract with the National Aeronautics and Space Administration.

This work is financially supported by the Grant-in-Aid for Scientific Research 19J22216 (S.Y.), 17K05384 and $20 \mathrm{H} 01946$ (Y.U.), 17J06407 (A.T.), $15 \mathrm{~K} 05030$ (M.I.), 18J01050 and 19K14759 (Y.T.). C.R. acknowledges the support from the CONICYT+PAI Convocatoria Nacional subvencion a instalacion en la academia convocatoria año 2017 PAI77170080.

Facilities: NuSTAR, Suzaku, XMM-Newton, Chandra 
Software: XCLUMPY (Tanimoto et al. 2019), HEAsoft 6.25, XSPEC (Arnaud 1996), NuSTARDAS, SAS 17.00 (Gabriel et al. 2004)

\section{REFERENCES}

Alexander, D. M., \& Hickox, R. C. 2012, NewAR, 56, 93, doi: 10.1016/j.newar.2011.11.003

Alonso-Herrero, A., Pereira-Santaella, M., Rieke, G. H., et al. 2013, ApJ, 765, 78, doi: 10.1088/0004-637X/765/2/78

Anders, E., \& Grevesse, N. 1989, GeoCoA, 53, 197, doi: 10.1016/0016-7037(89)90286-X

Armus, L., Mazzarella, J. M., Evans, A. S., et al. 2009, PASP, 121, 559, doi: 10.1086/600092

Arnaud, K. A. 1996, Astronomical Society of the Pacific Conference Series, Vol. 101, XSPEC: The First Ten Years, ed. G. H. Jacoby \& J. Barnes, 17

Asmus, D. 2019, MNRAS, 489, 2177, doi: $10.1093 / \mathrm{mnras} / \mathrm{stz} 2289$

Asmus, D., Gandhi, P., Hönig, S. F., Smette, A., \& Duschl, W. J. 2015, MNRAS, 454, 766, doi: 10.1093/mnras/stv1950

Asmus, D., Hönig, S. F., \& Gandhi, P. 2016, ApJ, 822, 109, doi: 10.3847/0004-637X/822/2/109

Asmus, D., Hönig, S. F., Gandhi, P., Smette, A., \& Duschl, W. J. 2014, MNRAS, 439, 1648, doi: 10.1093/mnras/stu041

Barro, G., Faber, S. M., Pérez-González, P. G., et al. 2013, ApJ, 765, 104, doi: 10.1088/0004-637X/765/2/104

Cowie, L. L., Songaila, A., Hu, E. M., \& Cohen, J. G. 1996, AJ, 112, 839, doi: 10.1086/118058

Dadina, M. 2008, A\&A, 485, 417, doi: 10.1051/0004-6361:20077569

Dasyra, K. M., Ho, L. C., Netzer, H., et al. 2011, ApJ, 740, 94, doi: 10.1088/0004-637X/740/2/94

Davies, R. L., Rich, J. A., Kewley, L. J., \& Dopita, M. A. 2014, MNRAS, 439, 3835, doi: 10.1093/mnras/stu234

Díaz-Santos, T., Armus, L., Charmandaris, V., et al. 2017, ApJ, 846, 32, doi: 10.3847/1538-4357/aa81d7

Draper, A. R., \& Ballantyne, D. R. 2012, ApJ, 751, 72, doi: 10.1088/0004-637X/751/1/72

Fukazawa, Y., Mizuno, T., Watanabe, S., et al. 2009, PASJ, 61, S17, doi: 10.1093/pasj/61.sp1.S17

Fukazawa, Y., Hiragi, K., Mizuno, M., et al. 2011, ApJ, 727, 19, doi: 10.1088/0004-637X/727/1/19

Furui, S., Fukazawa, Y., Odaka, H., et al. 2016, ApJ, 818, 164, doi: 10.3847/0004-637X/818/2/164
Gabriel, C., Denby, M., Fyfe, D. J., et al. 2004, Astronomical Society of the Pacific Conference Series, Vol. 314, The XMM-Newton SAS - Distributed Development and Maintenance of a Large Science Analysis System: A Critical Analysis, ed. F. Ochsenbein, M. G. Allen, \& D. Egret, 759

Gandhi, P., Horst, H., Smette, A., et al. 2009, A\&A, 502, 457, doi: 10.1051/0004-6361/200811368

García-Bernete, I., Ramos Almeida, C., Alonso-Herrero, A., et al. 2019, MNRAS, 486, 4917, doi: $10.1093 / \mathrm{mnras} / \mathrm{stz} 1003$

Ghisellini, G., Haardt, F., \& Matt, G. 1994, MNRAS, 267, 743, doi: 10.1093/mnras/267.3.743

Guainazzi, M., Matt, G., \& Perola, G. C. 2005, A\&A, 444, 119, doi: 10.1051/0004-6361:20053643

Harrison, F. A., Craig, W. W., Christensen, F. E., et al. 2013, ApJ, 770, 103, doi: 10.1088/0004-637X/770/2/103

Hönig, S. F., \& Beckert, T. 2007, MNRAS, 380, 1172, doi: 10.1111/j.1365-2966.2007.12157.x

Ichikawa, K., Packham, C., Ramos Almeida, C., et al. 2015, ApJ, 803, 57, doi: 10.1088/0004-637X/803/2/57

Ichikawa, K., Ricci, C., Ueda, Y., et al. 2019, ApJ, 870, 31, doi: 10.3847/1538-4357/aaef8f

Imanishi, M., Dudley, C. C., Maiolino, R., et al. 2007, ApJS, 171, 72, doi: 10.1086/513715

Imanishi, M., Dudley, C. C., \& Maloney, P. R. 2006, ApJ, 637, 114, doi: 10.1086/498391

Imanishi, M., Nakagawa, T., Ohyama, Y., et al. 2008, PASJ, 60, S489, doi: 10.1093/pasj/60.sp2.S489

Inami, H., Armus, L., Charmandaris, V., et al. 2013, ApJ, 777, 156, doi: 10.1088/0004-637X/777/2/156

Ishisaki, Y., Maeda, Y., Fujimoto, R., et al. 2007, PASJ, 59, 113, doi: 10.1093/pasj/59.sp1.S113

Jansen, F., Lumb, D., Altieri, B., et al. 2001, A\&A, 365, L1, doi: 10.1051/0004-6361:20000036

Kartaltepe, J. S., Sanders, D. B., Le Floc'h, E., et al. 2010, ApJ, 721, 98, doi: 10.1088/0004-637X/721/1/98

Kawamuro, T., Ueda, Y., Tazaki, F., Ricci, C., \& Terashima, Y. 2016, ApJS, 225, 14, doi: 10.3847/0067-0049/225/1/14

Kormendy, J., Bender, R., \& Cornell, M. E. 2011, Nature, 469, 374, doi: 10.1038/nature09694

Krolik, J. H., \& Begelman, M. C. 1988, ApJ, 329, 702, doi: 10.1086/166414 
Laurent, O., Mirabel, I. F., Charmandaris, V., et al. 2000, A\&A, 359, 887. https://arxiv.org/abs/astro-ph/0005376

Levenson, N. A., Weaver, K. A., Heckman, T. M., Awaki, H., \& Terashima, Y. 2004, ApJ, 602, 135, doi: $10.1086 / 380836$

Liu, Y., \& Li, X. 2014, ApJ, 787, 52, doi: 10.1088/0004-637X/787/1/52

Madau, P., \& Dickinson, M. 2014, ARA\&A, 52, 415, doi: 10.1146/annurev-astro-081811-125615

Madsen, K. K., Beardmore, A. P., Forster, K., et al. 2017, AJ, 153, 2, doi: 10.3847/1538-3881/153/1/2

Madsen, K. K., Harrison, F. A., Markwardt, C. B., et al. 2015, ApJS, 220, 8, doi: 10.1088/0067-0049/220/1/8

Magdziarz, P., \& Zdziarski, A. A. 1995, MNRAS, 273, 837, doi: 10.1093/mnras/273.3.837

Marinucci, A., Bianchi, S., Nicastro, F., Matt, G., \& Goulding, A. D. 2012, ApJ, 748, 130, doi: 10.1088/0004-637X/748/2/130

Mitsuda, K., Bautz, M., Inoue, H., et al. 2007, PASJ, 59, S1, doi: 10.1093/pasj/59.sp1.S1

Mullaney, J. R., Alexander, D. M., Goulding, A. D., \& Hickox, R. C. 2011, MNRAS, 414, 1082, doi: 10.1111/j.1365-2966.2011.18448.x

Nandra, K., O'Neill, P. M., George, I. M., \& Reeves, J. N. 2007, MNRAS, 382, 194, doi: 10.1111/j.1365-2966.2007.12331.x

Nenkova, M., Sirocky, M. M., Ivezić, Ž., \& Elitzur, M. 2008a, ApJ, 685, 147, doi: 10.1086/590482

Nenkova, M., Sirocky, M. M., Nikutta, R., Ivezić, Ž., \& Elitzur, M. 2008b, ApJ, 685, 160, doi: 10.1086/590483

Oda, S., Tanimoto, A., Ueda, Y., et al. 2017, ApJ, 835, 179, doi: $10.3847 / 1538-4357 / 835 / 2 / 179$

Ogawa, S., Ueda, Y., Tanimoto, A., \& Yamada, S. 2020, ApJ, submitted

Ogawa, S., Ueda, Y., Yamada, S., Tanimoto, A., \& Kawaguchi, T. 2019, ApJ, 875, 115, doi: 10.3847/1538-4357/ab0e08

Reines, A. E., \& Volonteri, M. 2015, ApJ, 813, 82, doi: 10.1088/0004-637X/813/2/82

Ricci, C., Ueda, Y., Ichikawa, K., et al. 2014, A\&A, 567, A142, doi: 10.1051/0004-6361/201322701

Ricci, C., Trakhtenbrot, B., Koss, M. J., et al. 2017a, Nature, 549, 488, doi: 10.1038/nature23906

Ricci, C., Bauer, F. E., Treister, E., et al. 2017b, MNRAS, 468, 1273, doi: 10.1093/mnras/stx173

Ricci, C., Trakhtenbrot, B., Koss, M. J., et al. 2017c, ApJS, 233, 17, doi: 10.3847/1538-4365/aa96ad

Rivers, E., Risaliti, G., Walton, D. J., et al. 2015, ApJ, 804, 107, doi: 10.1088/0004-637X/804/2/107
Romero-Cañizales, C., Alberdi, A., Ricci, C., et al. 2017, MNRAS, 467, 2504, doi: 10.1093/mnras/stx224

Sanders, D. B., Mazzarella, J. M., Kim, D. C., Surace, J. A., \& Soifer, B. T. 2003, AJ, 126, 1607, doi: $10.1086 / 376841$

Sanders, D. B., \& Mirabel, I. F. 1996, ARA\&A, 34, 749, doi: 10.1146/annurev.astro.34.1.749

Sanders, D. B., Soifer, B. T., Elias, J. H., Neugebauer, G., \& Matthews, K. 1988, ApJL, 328, L35, doi: $10.1086 / 185155$

Shangguan, J., Ho, L. C., Li, R., et al. 2019, ApJ, 870, 104, doi: 10.3847/1538-4357/aaf21a

Singh, V., Risaliti, G., Braito, V., \& Shastri, P. 2012, MNRAS, 419, 2089, doi: 10.1111/j.1365-2966.2011.19861.x

Smith, R. K., Brickhouse, N. S., Liedahl, D. A., \& Raymond, J. C. 2001, ApJL, 556, L91, doi: $10.1086 / 322992$

Stierwalt, S., Armus, L., Surace, J. A., et al. 2013, ApJS, 206, 1, doi: 10.1088/0067-0049/206/1/1

Tadaki, K.-i., Kodama, T., Tanaka, I., et al. 2014, ApJ, 780, 77, doi: 10.1088/0004-637X/780/1/77

Tanaka, I., Yagi, M., \& Taniguchi, Y. 2017, PASJ, 69, 90, doi: 10.1093/pasj/psx100

Tanimoto, A., Ueda, Y., Kawamuro, T., et al. 2018, ApJ, 853, 146, doi: 10.3847/1538-4357/aaa47c

Tanimoto, A., Ueda, Y., Odaka, H., et al. 2020, ApJ, submitted

—. 2019, ApJ, 877, 95, doi: 10.3847/1538-4357/ab1b20

Teng, S. H., Rigby, J. R., Stern, D., et al. 2015, ApJ, 814, 56, doi: 10.1088/0004-637X/814/1/56

Toba, Y., Ueda, Y., Matsuoka, K., et al. 2019, MNRAS, 484, 196, doi: 10.1093/mnras/sty3523

Tristram, K. R. W., Burtscher, L., Jaffe, W., et al. 2014, A\&A, 563, A82, doi: 10.1051/0004-6361/201322698

Ueda, Y., Akiyama, M., Hasinger, G., Miyaji, T., \& Watson, M. G. 2014, ApJ, 786, 104, doi: 10.1088/0004-637X/786/2/104

Ueda, Y., Akiyama, M., Ohta, K., \& Miyaji, T. 2003, ApJ, 598, 886, doi: 10.1086/378940

Ueda, Y., Eguchi, S., Terashima, Y., et al. 2007, ApJL, 664, L79, doi: 10.1086/520576

Vasudevan, R. V., \& Fabian, A. C. 2007, MNRAS, 381, 1235, doi: 10.1111/j.1365-2966.2007.12328.x

Véron-Cetty, M. P., \& Véron, P. 2003, A\&A, 412, 399, doi: 10.1051/0004-6361:20034225

Wada, K., \& Norman, C. A. 2002, ApJL, 566, L21, doi: 10.1086/339438

Wang, J. L., Xia, X. Y., Mao, S., et al. 2006, ApJ, 649, 722, doi: $10.1086 / 506902$ 
Weisskopf, M. C., Brinkman, B., Canizares, C., et al. 2002, PASP, 114, 1, doi: 10.1086/338108

Willingale, R., Starling, R. L. C., Beardmore, A. P., Tanvir, N. R., \& O’Brien, P. T. 2013, MNRAS, 431, 394, doi: $10.1093 / \mathrm{mnras} / \mathrm{stt} 175$
Yamada, S., Ueda, Y., Oda, S., et al. 2018, ApJ, 858, 106, doi: 10.3847/1538-4357/aabacb

Yamada, S., Ueda, Y., Tanimoto, A., et al. 2019, ApJ, 876, 96, doi: 10.3847/1538-4357/ab14f0

Yang, H., Wang, J., \& Liu, T. 2015, ApJ, 799, 91, doi: 10.1088/0004-637X/799/1/91 